\title{
The sacral networks and neural pathways used to elicit lumbar motor rhythm in the rodent spinal cord
}

\author{
Meir Cherniak, Alex Etlin, Ido Strauss, Lili Anglister and Aharon Lev-Tov* \\ Department of Medical Neurobiology, Institute for Medical Research-Israel-Canada, IMRIC, The Hebrew University Medical School, Jerusalem, Israel
}

\section{Edited by:}

Brian R. Noga, University of Miami,

USA

Reviewed by:

Uner Tan, Cukurova University,

Turkey

Patrick John Whelan, University of

Calgary, Canada

Kristine C. Cowley, University of

Manitoba, Canada

*Correspondence:

Aharon Lev-Tov, Department of Medical Neurobiology, Institute for Medical Research - Israel-Canada, IMRIC, The Hebrew University

Medical School, P.O. Box 12272, Jerusalem 9112102, Israel

e-mail: aharon/@ekmd.huji.ac.il
Identification of neural networks and pathways involved in activation and modulation of spinal central pattern generators (CPGs) in the absence of the descending control from the brain is important for further understanding of neural control of movement and for developing innovative therapeutic approaches to improve the mobility of spinal cord injury patients. Activation of the hindlimb innervating segments by sacrocaudal (SC) afferent input and by specific application of neurochemicals to the sacral networks is feasible in the isolated spinal cord preparation of the newborn rat. Here we review our recent studies of sacral relay neurons with lumbar projections and evaluate their role in linking the sacral and thoracolumbar (TL) networks during different motor behaviors. Our major findings show that: (1) heterogeneous groups of dorsal, intermediate and ventral sacralneurons with ventral and lateral ascending funicular projections mediate the activation of the locomotor CPGs through sacral sensory input; and (2) rhythmic excitation of lumbar flexor motoneurons, produced by bath application of alpha-1 adrenoceptor agonists to the sacral segments is mediated exclusively by ventral clusters of sacral-neurons with lumbar projections through the ventral funiculus.

Keywords: sacrocaudal afferents, calcium imaging, spinal interneurons, ascending pathways, central pattern generators, locomotor, alpha1 adrenoceptors

\section{INTRODUCTION: THE THORACOLUMBAR LIMB-MOVING AND SACROCAUDAL BODY-STABILIZING RHYTHMOGENIC NETWORKS ARE TIGHTLY COUPLED}

Spinal neuronal networks known as central pattern generators (CPGs) produce the rhythmic patterned output required for coordinated movements such as swimming and stepping in many species of vertebrates including humans (for reviews see Alford et al., 2003; Kiehn, 2006; Hultborn and Nielsen, 2007; Frigon, 2012). The CPGs are controlled by descending supraspinal commands and can be modulated to produce different patterns and behaviors. However, the CPGs can be also activated and modulated in the absence of descending control from brain by afferent-input and neuroactive compounds. Indeed, recent clinical studies have shown that reactivation of the CPGs in spinal cord injury patients by afferent input is possible and that it improves the motor function and mobility of some of the patients (for review see Hubli and Dietz, 2013). Therefore, it is of particular interest and significance to elucidate the networks and pathways involved in activation and modulation of the CPGs in accessible experimental models. The pattern generating circuitry in rodents activates the limb, trunk, and axial muscles innervating-segments to produce stabilized and coordinated locomotion under different conditions. The rhythmogenic capacity of these networks is preserved in vitro, in the isolated spinal cord preparation of newborn rodents (e.g., Kudo and Yamada, 1987; Smith et al., 1988). Therefore, this preparation can be used as an ideal model to study the mechanisms and interactions of the body stabilizing and limb moving networks in the absence of supraspinal control. In previous studies we described a rhythmogenic network in the sacrocaudal (SC) segments of the neonatal rat spinal cord that controls the axial and tail musculature during various movements (Lev-Tov and Delvolvé, 2000; Lev-Tov et al., 2000; Delvolvé et al., 2001; Gabbay et al., 2002). The SC-rhythmogenic network could be activated in surgically detached sacral spinal segments by stimulation of sacrocaudal afferents (SCA; Lev-Tov et al., 2000; Delvolvé et al., 2001), by bath application of noradrenaline (NA) and NMDA (Gabbay and Lev-Tov, 2004) and by the alphaladrenoceptor agonist, methoxamine (Gabbay and Lev-Tov, 2004). Figure 1 shows that the SC rhythmogenic network is co-activated with the hindlimb CPGs in the isolated spinal cord of the neonatal rat in the absence and presence of bath-applied neurochemicals. Coordinated activation of the SC- and locomotor-CPGs could be also demonstrated in response to electrical stimulation of the ventromedial medulla (VMM; Blivis et al., 2007; e.g., Zaporozhets et al., 2004) or SCA in the isolated brainstem-spinal cord preparation (Figure 1A, see Blivis et al., 2007; e.g., Gordon et al., 2008).

Most of the studies of rodent rhythmogenesis used various combinations of NMDA and monoamines to produce the rhythm (reviewed in Miles and Sillar, 2011). Figure 1B shows that the NMDA/5HT induced rhythm in the sacral spinal segments is nearly abolished when the spinal cord is transected at the lumbosacral junction, while the $0.25-1 \mathrm{~Hz}$ lumbar rhythm produced in the presence of methoxamine disappears when the 
A

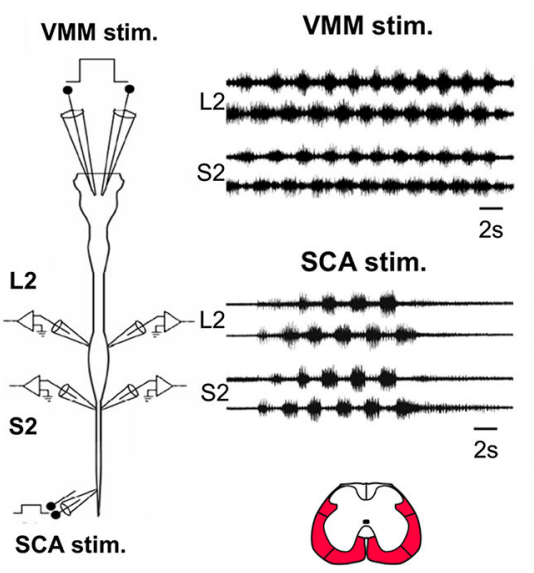

FIGURE 1 | Coordinated activation of the lumbar and sacral rhythmogenic networks. (A) Schematic illustration of the isolated brainstem spinal cord preparation of the neonatal rat (left). Stimulation electrodes are bilaterally attached to the ventromedial medulla (VMM) and to sacrocaudal afferents (SCA) entering though S4 or Co1 dorsal roots. Signals are recorded from left and right ( $L$ and $R$ ) ventral roots of L2 and S2. Right: Recordings of the rhythm produced by VMM (top) and SCA (bottom) stimulation from the left and right L2 and S2 ventral roots. VMM stimulation: $5 \mathrm{~ms}, 80$-pulse $4 \mathrm{~Hz}$ train at $1 \mathrm{~mA}$; SCA stimulation: $0.2 \mathrm{~ms}, 50$-pulse $4 \mathrm{~Hz}$ train at $25 \mu \mathrm{A}$ for the SCA. (B) The rhythm produced by bath application of $4 \mu \mathrm{M}$ NMDA and $10 \mu \mathrm{M} 5 \mathrm{HT}$ in the isolated spinal cord preparation of the newborn rat
B

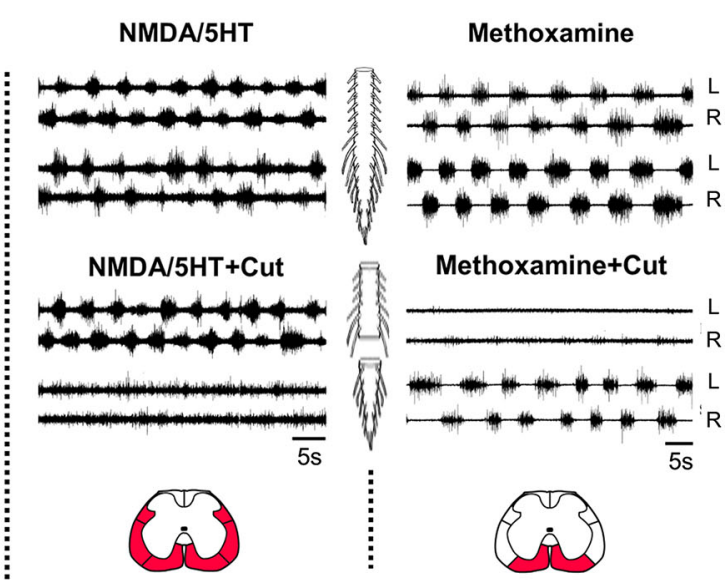

sacral segments are disconnected from the lumbar spinal cord (Figure 1C). These findings suggest that the lumbar-CPGs drive the sacral-CPGs in the presence of NMDA/5HT and that the sacral-CPGs drive the rostral-lumbar segments to produce rhythmic bursting in the presence of methoxamine (see also Gabbay and Lev-Tov, 2004). The coupling between the thoracolumbar (TL) and SC-CPGs during the rhythm induced by sensory and brainstem stimulation was found to be in the rostro-caudal direction (Bonnot et al., 2002; Lev-Tov and O'Donovan, 2009; Etlin et al., 2010; Lev-Tov et al., 2010; Strauss, 2011). On the other hand, caudo-rostral coupling between spinal networks is also well known in many vertebrates (see changes in the direction of propagation when switching from forward to backwards swimming in lampreys, Islam et al., 2006) including the neonatal rat spinal cord preparation during neurochemically induced motor rhythms (Falgairolle and Cazalets, 2007).

Our interest in the SC-network stems from two main reasons. First, it provides basic knowledge regarding stabilization of the body axis and the use of the tail musculature during rhythmic limb movements. Second, SC-interneurons may serve as important relays onto the locomotor generators during a nocifensive/escape type of behavior triggered by stimulation of SC dermatomes (Smith et al., 1988; Lev-Tov and Delvolvé, 2000; LevTov et al., 2000; Whelan et al., 2000; Delvolvé et al., 2001; Bonnot et al., 2002; Strauss and Lev-Tov, 2003). Moreover, the ability to activate the locomotor CPGs in the absence of descending control from the brain via sacral relays has a potential clinical significance for future treatments of spinal cord injury patients (see below). and recorded from the left and right L2 and S2 ventral roots, before (NMDA/5HT) and after transection of the cord through the lumbosacral junction (NMDA+5HT+Cut). (C) The rhythm produced by bath application of methoxamine $(100 \mu \mathrm{M})$ in the isolated spinal cord preparation of the newborn rat and recorded from the left and right L2 and S2 ventral roots, before (Methoxamine) and after (Methoxamine+Cut) transection of the cord through the lumbosacral junction. The red regions in the cross sections illustrated below denote the major white matter funiculi at the lumbosacral junction, linking the sacral and lumbar networks to produce coordinated rhythmic activity under the experimental conditions specified in $(\mathbf{A})$ (B) and (C), respectively.
More specifically, the capacity to produce the locomotor rhythm by SCA depends largely on synaptic activation of sacral relay neurons with lumbar projections (Strauss and Lev-Tov, 2003; Blivis et al., 2007; Etlin et al., 2010, 2013; Lev-Tov et al., 2010). The ability of sacral CPGs to produce an alternating leftright lumbar-flexor bursting in response to sacral application of methoxamine, also depends on activation of sacral relay neurons with rostral lumbar projections (Gabbay and Lev-Tov, 2004; as in Figure 1C). Here we briefly review our studies of the mediating relay neurons and the pathways involved in linking the sacral and lumbar networks under these conditions and discuss possible mechanisms and implications of our findings.

\section{I- ACTIVATION OF LOCOMOTOR CPGs BY SACROCAUDAL AFFERENT INPUT MEDIATION OF SCA-INDUCED LOCOMOTOR ACTIVITY BY SACRAL RELAY-NEURONS WITH LUMBAR PROJECTIONS}

Afferent input is a potent activator of the locomotor CPGs in the absence of the descending control from the brain in humans and experimental mammalian models (Grillner and Rossignol, 1978; Grillner and Zangger, 1979; Duysens and Pearson, 1980; Pearson and Rossignol, 1991; Prochazka et al., 1997; Dietz and Duysens, 2000; Dietz et al., 2002; Pearson, 2004; for reviews see Edgerton et al., 2008; Dietz, 2009). We showed that mechanical and radiant heat stimulation of SC dermatomes (Lev-Tov et al., 2000; and Blivis et al., 2007, respectively; e.g., Mandadi and Whelan, 2009) as well as electrical stimulation of SCA induces coordinated activation of the 
locomotor and SC-CPGs (Lev-Tov et al., 2000; Strauss and LevTov, 2003; Klein and Tresch, 2010; for the mouse see Whelan et al., 2000).

The SCA-induced locomotor rhythm in the newborn rat spinal cord, could be blocked by bathing the sacral segments in lowcalcium high-magnesium Kreb's saline, by selective sacral application of the non-NMDA receptor blocker CNQX (Strauss and Lev-Tov, 2003) or the mu-opioid agonist DAMGO (Blivis et al., 2007). Using surgical and pharmacological manipulations of the spinal cord, we found that the sacral relay neurons, activated by SCA to produce the locomotor rhythm, project to the lumbar segments through the ventral (VF), ventrolateral/lateral (VLF/LF) and dorsolateral (DLF) white matter funiculi (Strauss and LevTov, 2003; Etlin et al., 2010; Lev-Tov et al., 2010). These studies further showed that the capacity of SCA input to produce the locomotor rhythm depends not only on activation of sacral relay-neurons with direct projections to the lumbar CPGs, but also on serial recruitment of multi-funicular sacral-propriospinal neurons interposed between the second order neurons and the hindlimb-CPGs.

\section{SEGMENTAL AND SPATIAL DISTRIBUTION OF SACRAL RELAY NEURONS WITH LUMBAR PROJECTIONS}

Confocal imaging of sacral relay neurons with lumbar projections, back-labeled through cut VF, VLF/LF and DLF axon bundles at the lumbosacral junction, was used to map their segmental and spatial distribution, and lumbar projection patterns (Etlin et al., 2010; Lev-Tov et al., 2010). Figure 2 shows low-power projected confocal images of sacral relay-neurons in whole-mount transparent preparations of the spinal cord, labeled through the cut VF (Figure 2A) and the combined VLF/LF ("VLF", Figure 2B) axons at the left lumbosacral junction (see, respective scheme above each micrograph; also Etlin et al., 2010). These confocal micrographs demonstrate that most of the lumbar VF projections are crossed, and the majority of the lumbar VLF/LF projections are un-crossed (see also the schemes in A and B: crossed projections in blue, uncrossed projections in white). The DLF lumbar projections (not shown) were uncrossed. The back-labeled DLF-neurons were revealed mainly within the dorsal horn laminae, while most of the VF- and VLF/LF-neurons were in laminae VII, VIII and IX and the deep dorsal horn laminae (Etlin et al., 2010).

\section{SACROCAUDAL PRIMARY AFFERENTS AND INTRASPINAL NEURONS INNERVATE SACRAL RELAY-NEURONS WITH LUMBAR PROJECTIONS}

After mapping the distribution of sacral relay-neurons and their lumbar projection patterns, we examined whether these neurons are contacted by SCA, and whether these contacts are glutamatergic as predicted by Strauss and Lev-Tov (2003). Using backlabeling of sacral VF-neurons, anterograde labeling of SCA and immunostaining for vesicular glutamate transporters 1 and 2, we found that many VF-neurons are innervated by primary afferents immunoreactive for VGluT1, some VF-neurons were innervated by $\mathrm{VGluT2}^{+}$primary afferents, and large proportions of the VF-neurons received VGluT2 ${ }^{+}$contacts from intraspinal neurons (Etlin et al., 2013). These findings suggest (e.g., Alvarez et al., 2004; Liu et al., 2010; Scherrer et al., 2010; Rogoz et al., 2012) that the sacral VF-neurons can be activated directly by glutamatergic

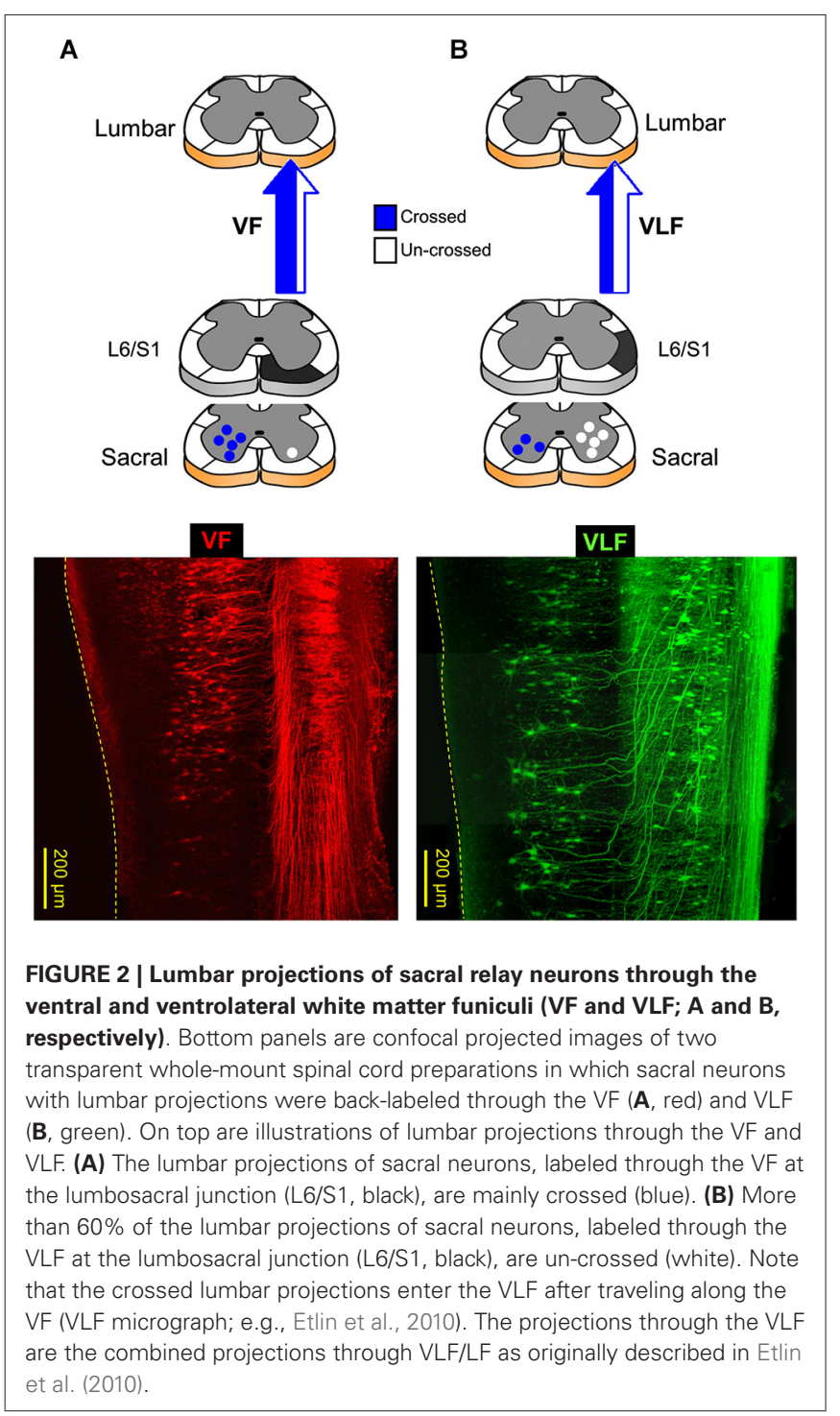

proprioceptive and nociceptive primary afferents and indirectly by glutamatergic-interneurons (Etlin et al., 2013).

\section{SACRAL VF-NEURONS SERVE AS AN IMPORTANT LINK BETWEEN SCA AND THE HINDLIMB-CPGs}

Etlin et al. (2010) have shown that bilateral interruption of the VF at the lumbosacral junction caused more severe interference to the lumbar rhythm produced by SCA stimulation than lesion of any other pair of white matter funiculi. They also showed that following multifunicular lesions that spared only one of the funiculi, the most regular and robust lumbar rhythm was obtained when the VF was left bilaterally intact (Etlin et al., 2010). Therefore, sacral relay-neurons projecting to the lumbar segments through the VF (VF-neurons) have been suggested to contribute more substantially than other sacral relay-neurons to activation of the lumbar-CPGs (Strauss and Lev-Tov, 2003; Etlin et al., 2010; Lev-Tov et al., 2010). These VF-neurons are innervated by glutamatergic SCA and intraspinal neurons (see above). The question raised next is whether VF-neurons are activated by 
SCA-stimulation, and if so, how their activity is related to the concomitant motor-output.

Figure 3 demonstrates calcium imaging of back-filled VFneurons and concurrent electrophysiological recordings of the motor-output produced in the rostral-lumbar and sacral segments of the isolated spinal cord during SCA stimulation (Figure 3A). Recordings were obtained from the ventral surface of the cord after prolonged back-loading with the calcium sensor (Calcium green dextran) through cut bundles of right VF-axons at the lumbosacral junction. The fluorescence changes $(\Delta \mathrm{F} / \mathrm{F})$ produced by calcium transients during the stimulus trains applied to SCA at two different intensities are shown in Figures 3B,C. The rhythmic $\Delta \mathrm{F} / \mathrm{F}$ of the imaged left VF-neuron during the higher-intensity stimulus train, exhibits a characteristic rhythmic pattern, in phase with the left ventral root bursting (Figure 3C). Our experiments revealed that more than $50 \%$ of the backlabeled VF-neurons could be activated by SCA stimulation. Fortyone percent of the activated VF-neurons exhibited tonic pattern, $18 \%$ showed pure rhythmic pattern, $34.5 \%$ had a tonic pattern with superimposed oscillations, and 6.2\% fired irregularly during the stimulus trains. Analysis of the 161 crossed-projecting VF-neurons that exhibited oscillatory drive (with and without background tonic activity), revealed that $\sim 70 \%$ of them were in phase with the ipsilateral-motor output and $~ 30 \%$ were in phase with the contralateral motor output (Etlin et al., 2013). We have suggested that VF-neurons with ipsilateral phase-preference are crossed-inhibitory and those with contralateral phase-preference are crossed-excitatory commissural neurons with lumbar projections (Etlin et al., 2013). This suggestion is based on the special pattern produced by sacral networks (alternating left-right rhythm and in-phase flexor-extensor activation at a given side of the sacral cord), and on studies of the phase preference of Vo commissural neurons (Lanuza et al., 2004) and lamina VIII GABAergic neurons (Wu et al., 2011).

Another interesting finding involves the relation between rhythmic bursting of the sacral CPG, the activity pattern of VFneurons, and lumbar motor-output. Figure 3D demonstrates that application of the NMDA-receptor blocker APV to the sacral segments reduces the activity of the sacral-CPGs without blocking the concomitant lumbar-rhythm. Under these conditions, the tonic drive of the imaged VF-neurons is not altered (compare Sacral APV to APV wash) while the oscillatory drive of the neurons is completely blocked in $\sim 60 \%$ of the cells with the combined rhythmic/tonic pattern and of the cells with the rhythmic pattern, and is markedly attenuated in the remaining $\sim 40 \%$ of these cells (Etlin et al., 2013). When APV is washed out, the sacralCPGs are vigorously activated; a strong rhythmic drive develops in the imaged neuron and the concomitant lumbar motor-output is strengthened significantly. All optical and electrophysiological activities are abolished in the presence of the non-NMDA receptor antagonist CNQX (Sacral CNQX), and reappear after washing the CNQX (CNQX wash). Collectively these findings suggest the following: (1) the ability of SCA stimulation to activate the VF-neurons and the CPGs depends on non-NMDA receptormediated synaptic transmission in the sacral segments; (2) activation of the lumbar CPGs by SCA stimulation can be obtained when the activity of the sacral CPGs is reduced or nearly blocked (see also Strauss and Lev-Tov, 2003), and when the drive of the recruited VF-neurons is purely tonic; and (3) the activities of VFneurons and the locomotor CPGs are maximized when the SC CPGs are activated. Thus, sacral VF-neurons are suggested to be a significant link between SCA and the locomotor CPGs.

\section{CAUDO-ROSTRAL COUPLING BETWEEN SACRAL AND ROSTRAL LUMBAR NETWORKS DURING ALPHA1-ADRENOCEPTOR AGONIST ACTIVATION OF THE SACRAL CPGs}

The spinal cord receives extensive noradrenergic innervation (e.g., Gabbay and Lev-Tov, 2004). Noradrenaline is known to initiate locomotor activity and modulate locomotor-like activity in a number of adult mammals (For review see Miles and Sillar, 2011). In the isolated spinal cord of neonatal rodents, NA produces a very slow rhythm with various irregularities (Kiehn et al., 1999; Cazalets and Bertrand, 2000; Sqalli-Houssaini and Cazalets, 2000). When applied to the sacral segments of the neonatal rat spinal cord, NA produces a short lasting (1-2 min) "fast" alternating left-right rhythm in the sacral and lumbar segments before transforming into a very slow non-locomotor rhythm (Gabbay and Lev-Tov, 2004). The "fast" NA rhythm was found to be blocked by alpha1 and not by alpha2 adrenoceptor antagonists (Gabbay et al., 2002). A "fast" (0.25-1 Hz) and robust alternating left-right rhythm could be produced and maintained in the isolated spinal cord of neonatal rats, in the presence of the alpha1-adrenoceptor agonist methoxamine (see Figures 1, 4). Our findings, that the methoxamine rhythm persists in the sacral segments and is blocked in the lumbar segments after transecting the spinal cord at the lumbosacral junction (Figure 1C, and Gabbay and Lev-Tov, 2004), suggest that the rhythm originates in the sacral segments of the spinal cord. The experiment shown in Figures 4B,C demonstrates that the $0.25-1 \mathrm{~Hz}$ methoxamine rhythm appears in both sacral and lumbar segments of the spinal cord only when methoxamine is added to the sacral but not to the TL spinal segments. Thus, there must be a potent coupling between the sacral CPGs and the rostral lumbar spinal segments.

What is the nature of the lumbar bursting produced by sacrally applied methoxamine? Gabbay and Lev-Tov (2004) showed that addition of methoxamine to the sacral segments produces rhythmic bursts in lumbar flexor but not in extensor motoneurons. They provided evidence suggesting that the sacral-CPGs do not activate the lumbar-CPGs in the presence of sacral methoxamine, but rather activate lumbar flexor-motoneurons and lumbar commissural-neurons (see Figure 9 in Gabbay and Lev-Tov, 2004, for the hypothetical organization of the circuitry). To study the link between the sacral-CPGs and the rostral lumbar segments under these conditions, we tried to determine the minimal anatomical configuration that is required to produce the $0.25-1 \mathrm{~Hz}$ rhythm in rostral lumbar motoneurons by sacrally applied methoxamine. Our most recent data (see abstracts by Cherniak et al., 2011; Roisman et al., 2014), revealed that: (1) the ability of methoxamine to produce the $0.25-1 \mathrm{~Hz}$ lumbar rhythm depends on intact connectivity between the TL and the first two sacral spinal segments (S1-S2); (2) the lumbar rhythm persists after removal of the dorsal aspect of the 
A

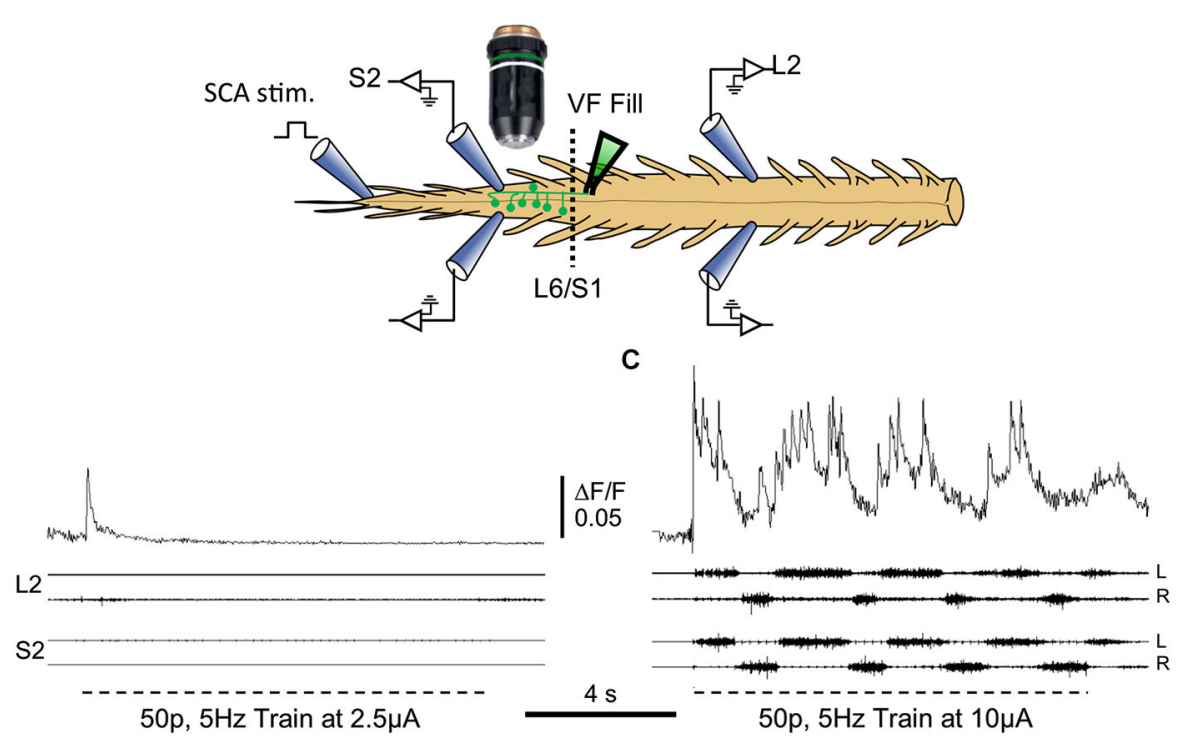

D
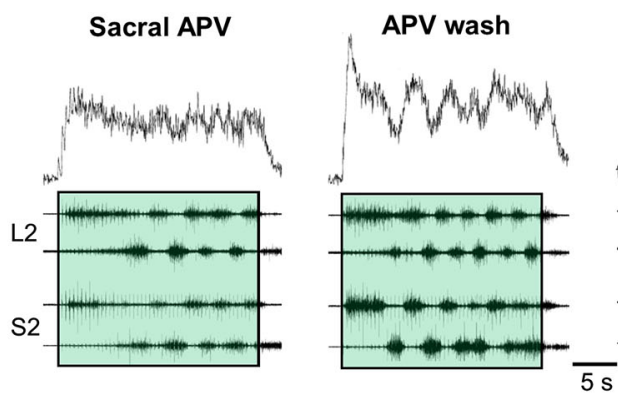

Sacral CNQX

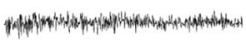
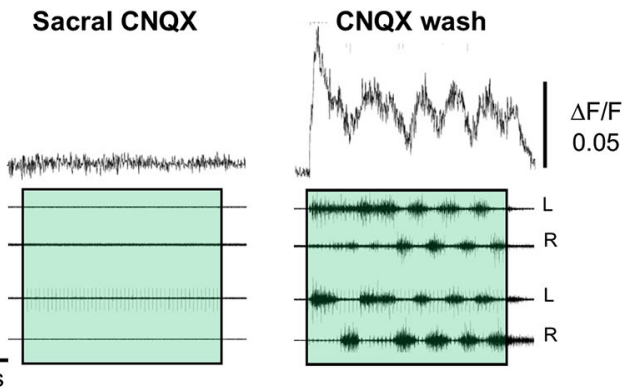

FIGURE 3 | Correlation between the activity of VF neurons and the motor output during SCA stimulation. (A) Illustration of the experimental set up used for simultaneous imaging of VF neurons and electrophysiological recordings of the motor output during the rhythm produced by SCA stimulation. Sacral relay neurons are loaded with calcium green dextran through the cut VF at the lumbosacral junction (L6/S1) in the isolated spinal cord preparation of the neonatal rat (VF fill). The preparation is mounted with ventral side up (in this example) in a dual chamber experimental bath (dashed line at the L6/S1 denotes the Vaseline barrier separating the two chambers). The motor output produced by stimulation of Co1 or S4 dorsal roots (SCA stimulation) is recorded from the left and right L2 and S2 ventral roots. Calcium transients produced in fluorescently labeled sacral VF-neurons are imaged simultaneously using epifluorescence microscopy with attached 14 bit CCD camera (for further details see Etlin et al., 2013). The difference image $(\triangle F / F)$ of imaged VF neurons and the simultaneous $L 2$ and S2 ventral root recordings during SCA stimulation under different conditions are superimposed in (B-D). The relations between the optical and electrophysiological signals are analyzed using the cross
Wavelet and cross Wavelet coherence methods (Mor and Lev-Tov, 2007; Etlin et al., 2013). (B,C) Top traces are the activity patterns imaged from a left $S 2 \mathrm{VF}$-neuron $(\Delta \mathrm{F} / \mathrm{F})$ back-labeled from the right $\mathrm{VF}$ with Calcium green dextran during $0.1 \mathrm{~ms}, 50-$ pulse, $5 \mathrm{~Hz}$ stimulus trains applied to the Co1 dorsal root at 2.5 and $10 \mu \mathrm{A}$ (B and $\mathbf{C}$, respectively). Below are the corresponding motor outputs (four bottom traces) recorded from the left and right ventral roots of S2 and L2, simultaneously with the optical signals. (D) The activity imaged from a VF-neuron ( $\Delta \mathrm{F} / \mathrm{F}$, upper records) and the motor output recorded from the left and right S2 and L2 ventral roots (four bottom records), during stimulation of the Co1 dorsal root, in the presence of $20 \mu \mathrm{M}$ of sacrally applied APV (Sacral APV) and 35 min after APV wash (APV wash). Sacral addition of $10 \mu \mathrm{M}$ CNOX under these conditions (Sacral (NOX) blocked the activity. The block is alleviated 60 min after CNOX wash (CNOX wash). Trains are confined within colored transparent rectangles. The oscillatory drive of the cells is markedly reduced by APV and is abolished by CNOX. For further details, see text and Etlin et al. (2013). Fifty-pulse $2.5 \mathrm{~Hz}$ stimulus trains were applied at $10 \mu \mathrm{A}$ to produce the rhythm. (B-D) Modified from Etlin et al. (2013). sacral cord down to the central canal, but not below it; and (3) the methoxamine-induced lumbar rhythm depends on ventral sacral-neurons projecting rostrally only through ventral funiculi. Following the latter finding, we imaged the activity of ventral clusters of back-labeled VF-neurons in the presence of methoxamine (Figure 4D) and studied their relation to the concomitant motor-output produced in the sacral- and lumbar-segments. The activity pattern of these ventral clusters of sacral VF-neurons in the presence of methoxamine was rhythmic in most cases with phase preference mainly to the ipsilateral motor output. Figure $4 \mathrm{E}$ shows the gradual appearance of rhythmic calcium transients in a left VF-neuron back-loaded with calcium green dextran and rhythmic bursting in sacral- and lumbar-segments in the presence of methoxamine. Regular optical and electrophysiological oscillatory signals developed after $10 \mathrm{~min}$ exposure to methoxamine. The $\Delta \mathrm{F} / \mathrm{F}$ of the left $\mathrm{VF}$ neuron imaged in 
A

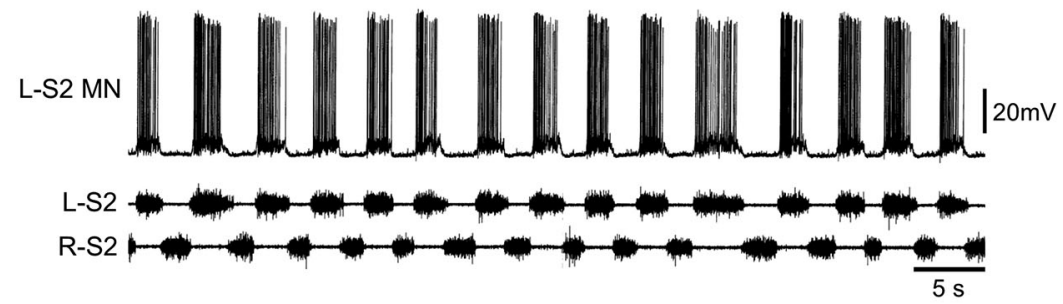

B
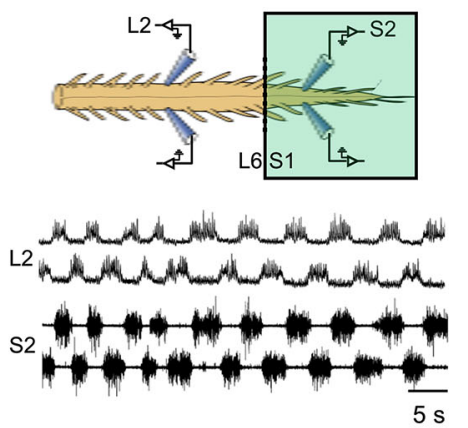

D

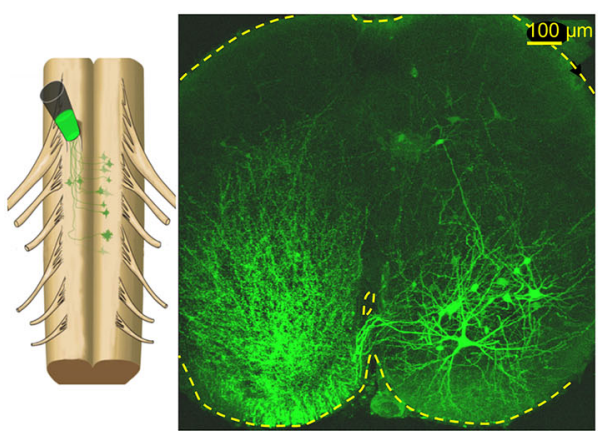

C
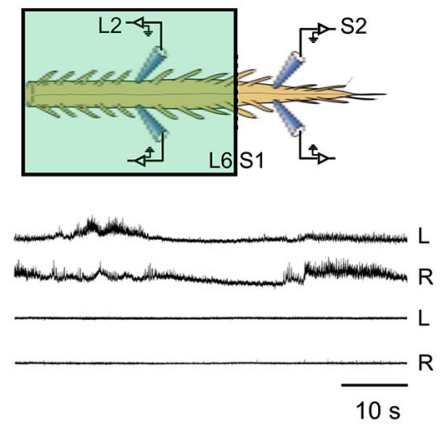

E
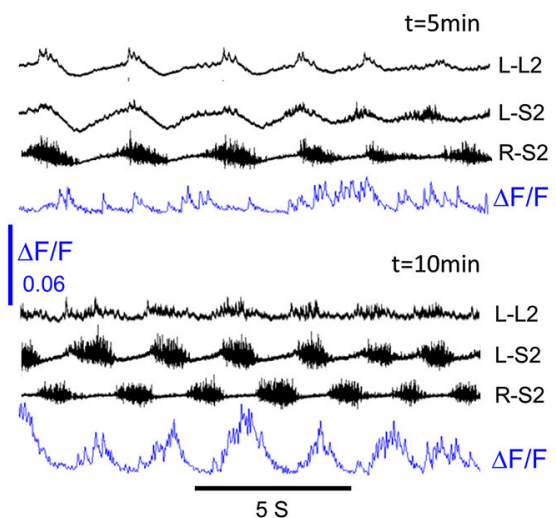

compartment (colored rectangle) of the experimental bath. The sacral rhythmic activity is blocked, while a slow rhythm appears in the lumbar segments. (D) Confocal micrograph of a $70 \mu \mathrm{m}$ cross section through the S2 spinal segment shows left sacral neurons back-loaded with fluorescein-dextran through the right VF at the lumbosacral junction (dye back-loading is illustrated on the left). (E) Imaging the activity developed in a ventral S2 VF-neuron back-loaded with Calcium green dextran and viewed from the ventral aspect of the isolated spinal cord (blue, $\Delta F / F$ ) and the concurrently recorded motor output from the left L2 and the left and right S2 ventral roots, 5 and $10 \mathrm{~min}$ after addition of methoxamine to the experimental bath. The

$0.1 \mathrm{~Hz}-10 \mathrm{Khz}$ ventral root recordings were not high-pass filtered to reveal the early subthreshold activity in L-L2. this experiment is in phase with the ipsilateral motor output (L-S2).

We suggest that sub-clusters of ventrally located sacralneurons mediate the generation of the rhythmic bursting in lumbar flexor-motoneurons by sacrally applied methoxamine. Studies of trans-synaptic labeling of sacral VF- and other sacral-interneurons, using GFP-encoded retrograde virus injection to hindlimb muscles (e.g., Hadas et al., 2014), suggest oligo-synaptic connectivity between sacral VF-neurons and rostral lumbar motoneurons. Additional experiments are needed to further clarify this issue and determine the functionality of this connectivity.

\section{CONCLUSIONS AND SUGGESTIONS}

In this manuscript, we focused on the mediating role of sacral-neurons in linking the sacral and lumbar networks during 
rhythmic motor activity produced by SCA input and by selective activation of sacral-neurons by alphal adrenoceptor agonists. While SCA stimulation activates successfully both the sacral and lumbar CPGs, sacrally applied methoxamine activates the sacral CPGs and thereby produces alternating left-right bursting in rostral lumbar motoneurons. The lumbar rhythmic bursting produced by SCA stimulation is mediated mainly by relay-neurons with direct and indirect multifunicular lumbar-projections, while the methoxamine-induced rhythm is mediated by sacral-neurons that project rostrally only through the VF. We provided evidence that the drive produced by the activated VF relay neurons and the pathways associated with them, may turn-on the locomotor pattern generators when the sacral afferents are stimulated, and stimulate lumbar flexor motoneurons in the presence of methoxamine. Thus, sub-populations of sacral VF-neurons are suggested to project to flexor motoneurons that are driven by the more excitable and dominant rostral lumbar oscillators (e.g., Cazalets et al., 1995; Kjaerulff and Kiehn, 1996; Cowley and Schmidt, 1997; Kremer and Lev-Tov, 1997; Bonnot et al., 2002; for review see Kiehn, 2006), and to the locomotor CPGs (Etlin et al., 2010, 2013). Modulation of the activity of these two sets of projections enables separate and/or simultaneous control on the frequency and power of the rhythmic lumbar output under different conditions (see Finkel et al., 2014), and thereby plays a significant role in shaping the final motor-output.

Further studies are required to clarify the role of short- and long-ascending propriospinal pathways from the sacral to the lumbar cord (Bras et al., 1988; Grottel et al., 1998; Dutton et al., 2006), and of lumbar collaterals of spinothalamic, spinocerebellar or spinoreticular pathways (Leah et al., 1988; Edgley and Grant, 1991; Yamada et al., 1991; Katter et al., 1996; Matsushita, 1999; Garifoli et al., 2006) in activation of the locomotor CPGs, and the exact mechanisms by which inter-enlargements coupling is achieved. It is also important to verify whether it is possible to extrapolate our findings in newborn rodent to the adult spinal cord, and to evaluate the potential clinical significance of the ability to activate and modulate the CPG action via sacral relay neurons in the absence of descending supraspinal control.

\section{ACKNOWLEDGMENTS}

This work was supported by grants from The Israel Science Foundation (ISF) 1591/08, 1930/08, 491/12 to Aharon Lev-Tov, and ISF 685/01 and MDA to Lili Anglister.

\section{REFERENCES}

Alford, S., Schwartz, E., and Viana di Prisco, G. (2003). The pharmacology of vertebrate spinal central pattern generators. Neuroscientist 9, 217-228. doi: 10 . 1177/1073858403009003014

Alvarez, F. J., Villalba, R. M., Zerda, R., and Schneider, S. P. (2004). Vesicular glutamate transporters in the spinal cord, with special reference to sensory primary afferent synapses. J. Comp. Neurol. 472, 257-280. doi: 10.1002/cne. 20012

Blivis, D., Mentis, G. Z., O’Donovan, M. J., and Lev-Tov, A. (2007). Differential effects of opioids on sacrocaudal afferent pathways and central pattern generators in the neonatal rat spinal cord. J. Neurophysiol. 97, 2875-2886. doi: 10. 1152/jn.01313.2006

Bonnot, A., Whelan, P. J., Mentis, G. Z., and O'Donovan, M. J. (2002). Spatiotemporal pattern of motoneuron activation in the rostral lumbar and the sacral segments during locomotor-like activity in the neonatal mouse spinal cord. J. Neurosci. 22:RC203.
Bras, H., Cavallari, P., and Jankowska, E. (1988). Demonstration of initial axon collaterals of cells of origin of the ventral spinocerebellar tract in the cat. J. Comp. Neurol. 273, 584-592. doi: 10.1002/cne.902730412

Cazalets, J. R., and Bertrand, S. (2000). Coupling between lumbar and sacral motor networks in the neonatal rat spinal cord. Eur. J. Neurosci. 12, 2993-3002. doi: 10. 1046/j.1460-9568.2000.00169.x

Cazalets, J. R., Borde, M., and Clarac, F. (1995). Localization and organization of the central pattern generator for hindlimb locomotion in newborn rat. J. Neurosci. 15, 4943-4951.

Cherniak, M., Etlin, A., Federman, N., Srauss, I., and Lev-Tov, A. (2011). Ventral horn clusters of sacral neurons are activated by $\alpha 1$ adrenoceptor agonists to produce "fast" rhythmic bursts in sacral and rostral lumbar motoneurons. J. Mol. Neurosci. 45(Suppl. 1), S24.

Cowley, K. C., and Schmidt, B. J. (1997). Regional distribution of the locomotor pattern-generating network in the neonatal rat spinal cord. J. Neurophysiol. 77, 247-259.

Delvolvé, I., Gabbay, H., and Lev-Tov, A. (2001). The motor output and behavior produced by rhythmogenic sacrocaudal networks in spinal cords of neonatal rats. J. Neurophysiol. 85, 2100-2110.

Dietz, V. (2009). Body weight supported gait training: from laboratory to clinical setting. Brain Res. Bull. 78, I-VI. doi: 10.1016/S0361-9230(08) 00410-3

Dietz, V., and Duysens, J. (2000). Significance of load receptor input during locomotion: a review. Gait Posture 11, 102-110. doi: 10.1016/S0966-6362(99) 00052-1

Dietz, V., Müller, R., and Colombo, G. (2002). Locomotor activity in spinal man: significance of afferent input from joint and load receptors. Brain 125, 26262634. doi: 10.1093/brain/awf273

Dutton, R. C., Carstens, M. I., Antognini, J. F., and Carstens, E. (2006). Long ascending propriospinal projections from lumbosacral to upper cervical spinal cord in the rat. Brain Res. 1119, 76-85. doi: 10.1016/j.brainres.2006. 08.063

Duysens, J., and Pearson, K. G. (1980). Inhibition of flexor burst generation by loading ankle extensor muscles in walking cats. Brain Res. 187, 321-332. doi: 10. 1016/0006-8993(80)90206-1

Edgerton, V. R., Courtine, G., Gerasimenko, Y. P., Lavrov, I., Ichiyama, R. M., Fong, A. J., et al. (2008). Training locomotor networks. Brain Res. Rev. 57, 241-254. doi: 10.1016/j.brainresrev.2007.09.002

Edgley, S. A., and Grant, G. M. (1991). Inputs to spinocerebellar tract neurons located in stilling's nucleus in the sacral segments of the rat spinal cord. J. Comp. Neurol. 305, 130-138. doi: 10.1002/cne.903050112

Etlin, A., Blivis, D., Ben-Zwi, M., and Lev-Tov, A. (2010). Long and short multifunicular projections of sacral neurons are activated by sensory input to produce locomotor activity in the absence of supraspinal control. J. Neurosci. 30, 10324 10336. doi: 10.1523/JNEUROSCI.1208-10.2010

Etlin, A., Finkel, E., Mor, Y., O’Donovan, M. J., Anglister, L., and Lev-Tov, A. (2013). Characterization of sacral interneurons that mediate activation of locomotor pattern generators by sacrocaudal afferent input. J. Neurosci. 33, 734-747. doi: 10.1523/JNEUROSCI.4390-12.2013

Falgairolle, M., and Cazalets, J. R. (2007). Metachronal coupling between spinal neuronal networks during locomotor activity in newborn rat. J. Physiol. Lond. 580, 87-102. doi: 10.1113/jphysiol.2006.115709

Finkel, E., Etlin, A., Cherniak, M., Mor, Y., Lev-Tov, A., and Anglister, L. (2014). Neuroanatomical basis for cholinergic modulation of locomotor networks by sacral relay neurons with ascending lumbar projections. J. Comp. Neurol. 522, 3437-3455. doi: 10.1002/cne.23613

Frigon, A. (2012). Central pattern generators of the mammalian spinal cord. Neuroscientist 18, 56-69. doi: 10.1177/1073858410396101

Gabbay, H., Delvolvé, I., and Lev-Tov, A. (2002). Pattern generation in caudallumbar and sacrococcygeal segments of the neonatal rat spinal cord. J. Neurophysiol. 88, 732-739.

Gabbay, H., and Lev-Tov, A. (2004). Alpha-1 adrenoceptor agonists generate a "fast" NMDA receptor-independent motor rhythm in the neonatal rat spinal cord. J. Neurophysiol. 92, 997-1010. doi: 10.1152/jn.00205.2004

Garifoli, A., Maci, T., Perciavalle, V., and Perciavalle, V. (2006). Organization of bilateral spinal projections to the lateral reticular nucleus of the rat. Arch. Ital. Biol. 144, 145-157.

Gordon, I. T., Dunbar, M. J., Vanneste, K. J., and and Whelan, P. J. (2008). Interaction between developing spinal locomotor networks in the 
neonatal mouse. J. Neurophysiol. 100, 117-128. doi: 10.1152/jn.00829. 2007

Grillner, S., and Rossignol, S. (1978). On the initiation of the swing phase of locomotion in chronic spinal cats. Brain Res. 146, 269-277. doi: 10.1016/00068993(78)90973-3

Grillner, S., and Zangger, P. (1979). On the central generation of locomotion in the low spinal cat. Exp. Brain Res. 34, 241-261. doi: 10.1007/BF00235671

Grottel, K., Krutki, P., and Mrówczyński, W. (1998). Triple projections of neurones located in S1 and S2 segments of the cat spinal cord to the C6 segment, the cerebellum and the reticular formation. Exp. Physiol. 83, 737-746.

Hadas, Y., Etlin, A., Falk, H., Avraham, O., Kobiler, O., Panet, A., et al. (2014). A 'tool box' for deciphering neuronal circuits in the developing chick spinal cord. Nucleic Acids Res. doi: 10.1093/nar/gku750. [Epub ahead of print].

Hubli, M., and Dietz, V. (2013). The physiological basis of neurorehabilitationlocomotor training after spinal cord injury. J. Neuroeng. Rehabil. 10:5. doi: 10. 1186/1743-0003-10-5

Hultborn, H., and Nielsen, J. B. (2007). Spinal control of locomotion-from cat to man. Acta Physiol. (Oxf) 189, 111-121. doi: 10.1111/j.1748-1716.2006.01651.x

Islam, S. S., Zelenin, P. V., Orlovsky, G. N., Grillner, S., and Deliagina, T. G. (2006). Pattern of motor coordination underlying backward swimming in the lamprey. J. Neurophysiol. 96, 451-460. doi: 10.1152/jn.01277.2005

Katter, J. T., Dado, R. J., Kostarczyk, E., and Giesler, G. J. Jr. (1996). Spinothalamic and spinohypothalamic tract neurons in the sacral spinal cord of rats. I. Locations of antidromically identified axons in the cervical cord and diencephalon. J. Neurophysiol. 75, 2581-2605.

Kiehn, O. (2006). Locomotor circuits in the mammalian spinal cord. Annu. Rev. Neurosci. 29, 279-306. doi: 10.1146/annurev.neuro.29.051605.112910

Kiehn, O., Sillar, K. T., Kjaerulff, O., and McDearmid, J. R. (1999). Effects of noradrenaline on locomotor rhythm-generating networks in the isolated neonatal rat spinal cord. J. Neurophysiol. 82, 741-746.

Kjaerulff, O., and Kiehn, O. (1996). Distribution of networks generating and coordinating locomotor activity in the neonatal rat spinal cord in vitro: a lesion study. J. Neurosci. 16, 5777-5794.

Klein, D. A., and Tresch, M. C. (2010). Specificity of intramuscular activation during rhythms produced by spinal patterning systems in the in vitro neonatal rat with hindlimb attached preparation. J. Neurophysiol. 104, 2158-2168. doi: 10. 1152/jn.00477.2010

Kremer, E., and Lev-Tov, A. (1997). Localization of the spinal network associated with generation of hindlimb locomotion in the neonatal rat and organization of its transverse coupling system. J. Neurophysiol. 77, 1155-1170.

Kudo, N., and Yamada, T. (1987). N-methyl-D,L-aspartate-induced locomotor activity in a spinal cord-hindlimb muscles preparation of the newborn rat studied in vitro. Neurosci. Lett. 75, 43-48. doi: 10.1016/0304-3940(87)90072-3

Lanuza, G. M., Gosgnach, S., Pierani, A., Jessell, T. M., and Goulding, M. (2004). Genetic identification of spinal interneurons that coordinate left-right locomotor activity necessary for walking movements. Neuron 42, 375-386. doi: 10 . 1016/S0896-6273(04)00249-1

Leah, J., Menétrey, D., and de Pommery, J. (1988). Neuropeptides in long ascending spinal tract cells in the rat: evidence for parallel processing of ascending information. Neuroscience 24, 195-207. doi: 10.1016/0306-4522(88)90323-5

Lev-Tov, A., and Delvolvé, I. (2000). Pattern generation in non-limb moving segments of the mammalian spinal cord. Brain Res. Bull. 53, 671-675. doi: 10. 1016/S0361-9230(00)00400-7

Lev-Tov, A., Delvolvé, I., and Kremer, E. (2000). Sacrocaudal afferents induce rhythmic efferent bursting in isolated spinal cords of neonatal rats. J. Neurophysiol. 83, 888-894.

Lev-Tov, A., Etlin, A., and Blivis, D. (2010). Sensory-induced activation of pattern generators in the absence of supraspinal control. Ann. N Y Acad. Sci. 1198, $54-$ 62. doi: 10.1111/j.1749-6632.2009.05424.x

Lev-Tov, A., and O'Donovan, M. (2009). "Spinal cord: neonatal circuits," in Encyclopedia of Neuroscience, ed L. R. Squire, Editor-in-Chief (Oxford: Academic Press), 61-65.

Liu, Y., Abdel Samad, O., Zhang, L., Duan, B., Tong, Q., Lopes, C., et al. (2010). VGLUT2-dependent glutamate release from nociceptors is required to sense pain and suppress itch. Neuron 68, 543-556. doi: 10.1016/j.neuron.2010.09.008

Mandadi, S., and Whelan, P. J. (2009). A new method to study sensory modulation of locomotor networks by activation of thermosensitive cutaneous afferents using a hindlimb attached spinal cord preparation. J. Neurosci. Methods 182, 255-259. doi: 10.1016/j.jneumeth.2009.06.011
Matsushita, M. (1999). Projections from lowest lumbar and sacral caudal segments to the cerebellar nuclei in the rat, studied by anterograde axonal tracing. J. Comp. Neurol. 404, 21-32. doi: 10.1002/(SICI)1096-9861(19990201)404:1<21::AID$\mathrm{CNE} 2>3.0 . \mathrm{CO} ; 2-7$

Miles, G. B., and Sillar, K. T. (2011). Neuromodulation of vertebrate locomotor control networks. Physiology (Bethesda) 26, 393-411. doi: 10.1152/physiol. 00013.2011

Mor, Y., and Lev-Tov, A. (2007). Analysis of rhythmic patterns produced by spinal neural networks. J. Neurophysiol. 98, 2807-2817. doi: 10.1152/jn.00740.2007

Pearson, K. G. (2004). Generating the walking gait: role of sensory feedback. Prog. Brain Res. 143, 123-129. doi: 10.1016/S0079-6123(03)43012-4

Pearson, K. G., and Rossignol, S. (1991). Fictive motor patterns in chronic spinal cats. J. Neurophysiol. 66, 1874-1887.

Prochazka, A., Gillard, D., and Bennett, D. J. (1997). Positive force feedback control of muscles. J. Neurophysiol. 77, 3226-3236.

Rogoz, K., Lagerström, M. C., Dufour, S., and Kullander, K. (2012). VGLUT2dependent glutamatergic transmission in primary afferents is required for intact nociception in both acute and persistent pain modalities. Pain 153, 1525-1536. doi: 10.1016/j.pain.2012.04.017

Roisman, R., Cherniak, M., and Lev-Tov, A. (2014). Caudo-rostral coupling between alpha-1 adrenoceptor activated cluster of sacral interneurons and rostral lumbar motoneurons. J. Mol. Neurosci. 53(Suppl. 1), S107-S108.

Scherrer, G., Low, S. A., Wang, X., Zhang, J., Yamanaka, H., Urban, R., et al. (2010). VGLUT2 expression in primary afferent neurons is essential for normal acute pain and injury-induced heat hypersensitivity. Proc. Natl. Acad. Sci. U S A 107, 22296-22301. doi: 10.1073/pnas.1013413108

Smith, J. C., Feldman, J. L., and Schmidt, B. J. (1988). Neural mechanisms generating locomotion studied in mammalian brain stem-spinal cord in vitro. FASEB J. 2, 2283-2288.

Sqalli-Houssaini, Y., and Cazalets, J. R. (2000). Noradrenergic control of locomotor networks in the in vitro spinal cord of the neonatal rat. Brain Res. 852, 100-109. doi: 10.1016/S0006-8993(99)02219-2

Strauss, I. (2011). Coupling Between Rhythmogenic Networks in the Isolated Spinal Cord of the Neonatal Rat. PhD thesis, Jerusalem: Hebrew University.

Strauss, I., and Lev-Tov, A. (2003). Neural pathways between sacrocaudal afferents and lumbar pattern generators in neonatal rats. J. Neurophysiol. 89, 773-784. doi: $10.1152 /$ jn.00716.2002

Whelan, P., Bonnot, A., and O'Donovan, M. J. (2000). Properties of rhythmic activity generated by the isolated spinal cord of the neonatal mouse. J. Neurophysiol. 84, 2821-2833.

Wu, L., Sonner, P. M., Titus, D. J., Wiesner, E. P., Alvarez, F. J., and ZiskindConhaim, L. (2011). Properties of a distinct subpopulation of GABAergic commissural interneurons that are part of the locomotor circuitry in the neonatal spinal cord. J. Neurosci. 31, 4821-4833. doi: 10.1523/JNEUROSCI.4764-10.2011

Yamada, J., Shirao, K., Kitamura, T., and Sato, H. (1991). Trajectory of spinocerebellar fibers passing through the inferior and superior cerebellar peduncles in the rat spinal cord: a study using horseradish peroxidase with pedunculotomy. J. Comp. Neurol. 304, 147-160. doi: 10.1002/cne.903040111

Zaporozhets, E., Cowley, K. C., and Schmidt, B. J. (2004). A reliable technique for the induction of locomotor-like activity in the in vitro neonatal rat spinal cord using brainstem electrical stimulation. J. Neurosci. Methods 139, 33-41. doi: 10. 1016/j.jneumeth.2004.04.009

Conflict of Interest Statement: The authors declare that the research was conducted in the absence of any commercial or financial relationships that could be construed as a potential conflict of interest.

Received: 16 June 2014; accepted: 11 November 2014; published online: 03 December 2014.

Citation: Cherniak M, Etlin A, Strauss I, Anglister L and Lev-Tov A (2014) The sacral networks and neural pathways used to elicit lumbar motor rhythm in the rodent spinal cord. Front. Neural Circuits 8:143. doi: 10.3389/fncir.2014.00143

This article was submitted to the journal Frontiers in Neural Circuits.

Copyright (c) 2014 Cherniak, Etlin, Strauss, Anglister and Lev-Tov. This is an openaccess article distributed under the terms of the Creative Commons Attribution License (CC BY). The use, distribution and reproduction in other forums is permitted, provided the original author(s) or licensor are credited and that the original publication in this journal is cited, in accordance with accepted academic practice. No use, distribution or reproduction is permitted which does not comply with these terms. 